\title{
Distribution of Depression and Suicidality in a Psoriasis Clinical Trial Population
}

\author{
Steven R. Feldman,' Susan Harris, ${ }^{2}$ Shipra Rastogi, ${ }^{3}$ Robert J. Israel ${ }^{2}$
}

'Wake Forest University School of Medicine, Winston-Salem, NC; ${ }^{2}$ Valeant Pharmaceuticals North America LLC, Bridgewater, NJ; ${ }^{3}$ Ortho Dermatologics, Bridgewater, NJ

\section{SYNOPSIS}

- Patients with psoriasis have an increased risk of depression and suicidal ideation and behavior (SIB) ${ }^{1.2}$

- Brodalumab is a fully human anti-interleukin-17 receptor $A$ monoclonal antibody that antagonizes the action of specific inflammatory cytokines involved in psoriasis

- One multicenter, randomized, placebo-controlled phase 3 trial (AMAGINE-I), 2 multicenter, randomized, placeboand active comparator-controlled phase 3 trials (AMAGINE-2/-3), and one phase 2 trial demonstrated the efficacy

- All regions involved in the trials reported baseline rates of depression and SIB

- Rates of SIB events were low throughout all trials (range, 0 to 0.77 per 100 patient-years [PY])

\section{OBJECTIVE}

- This analysis evaluated rates of depression adverse events (AEs) and SIB in patients participating in one phase 2 and three phase 3 clinical trials of brodalumab

\section{METHODS}

Study design

- Pooled data for all trials in patients who received any dose of brodalumab are included

- Of note, the brodalumab trials had no exclusions based on the presence or history of psychiatric disorders or substance abuse

Endpoints/Assessments

- Rates of depression AEs and SIB (intentional self-injury, suicidal behavior, suicide attempt, and completed suicide) in the United States, Canada, Europe, Australia, and Russia were assessed at baseline and throughout the trials

\section{RESULTS}

Patient demographics and baseline disease characteristics

- A total of 4464 patients received brodalumab, with cumulative exposure times of $3672.6 \mathrm{PY}$ in the US ( $\mathrm{n}=1937)$ 1473.4 PY in Canada $(n=631)$, 3492.7 PY in Europe $(n=165 I), 388.8$ PY in Australia $(n=180)$, and I34.4 PY in Russia $(n=65)$

Safety

- At baseline, depression was observed across most study regions, which is representative of the psoriasis population (Figure I)

Figure I. Incidence of depression at baseline across geographic regions in patients who received any dose of brodalumab in any of the 4 trials.

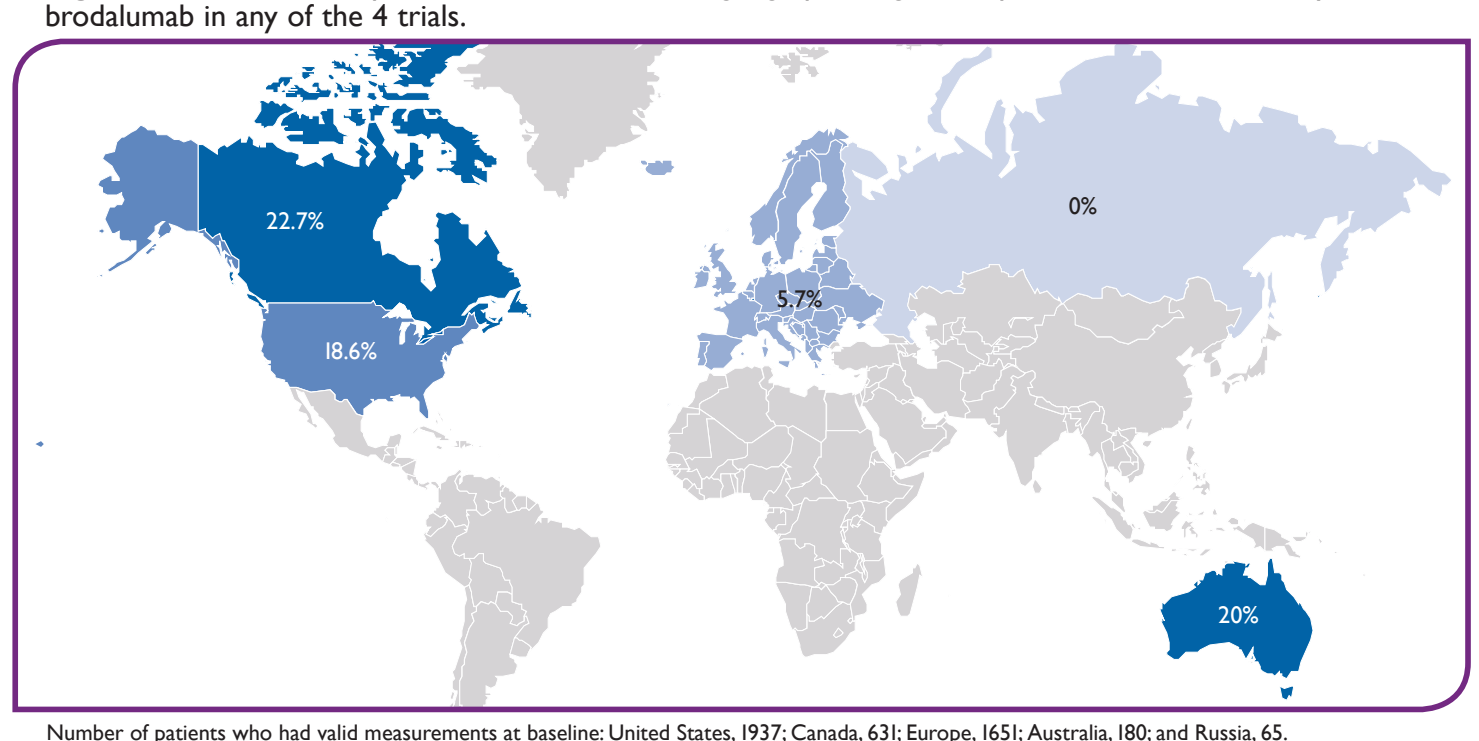

- Depression AE rate in PY from the first dose of brodalumab through end of study was also consistent across regions (Figure 2)

Figure 2. Depression adverse event rate in patient-years from the first dose of brodalumab through end of study,

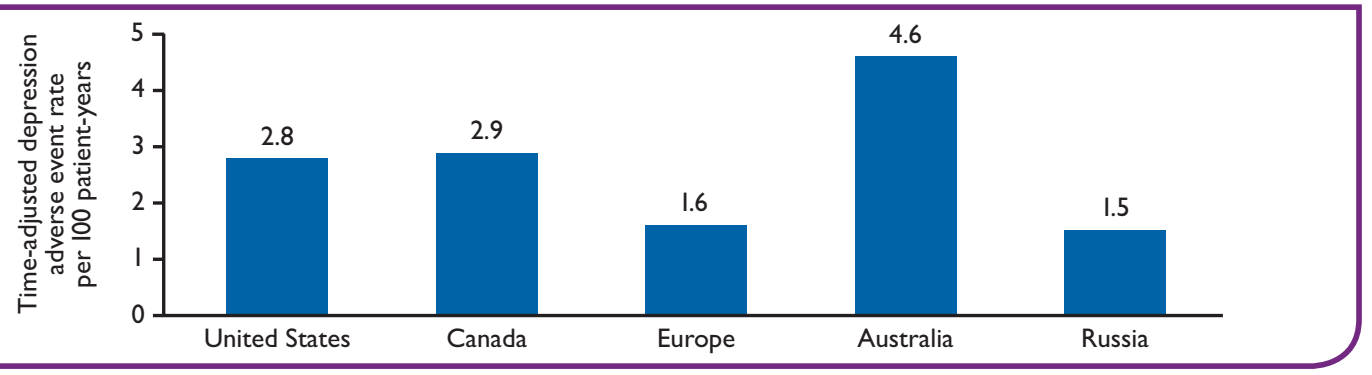

Total patient-year exposure for each region: United States, 3680.9; Canada, 1473.5; Europe, 3496.3; Australia, 388.9; and Russia, 134.4.
- SIB was observed at baseline across all countries involved in the 4 trials (Figure 3)

Figure 3. Incidence of baseline SIB across geographic regions in patients who received any dose of brodalumab in any of the 4 trials.

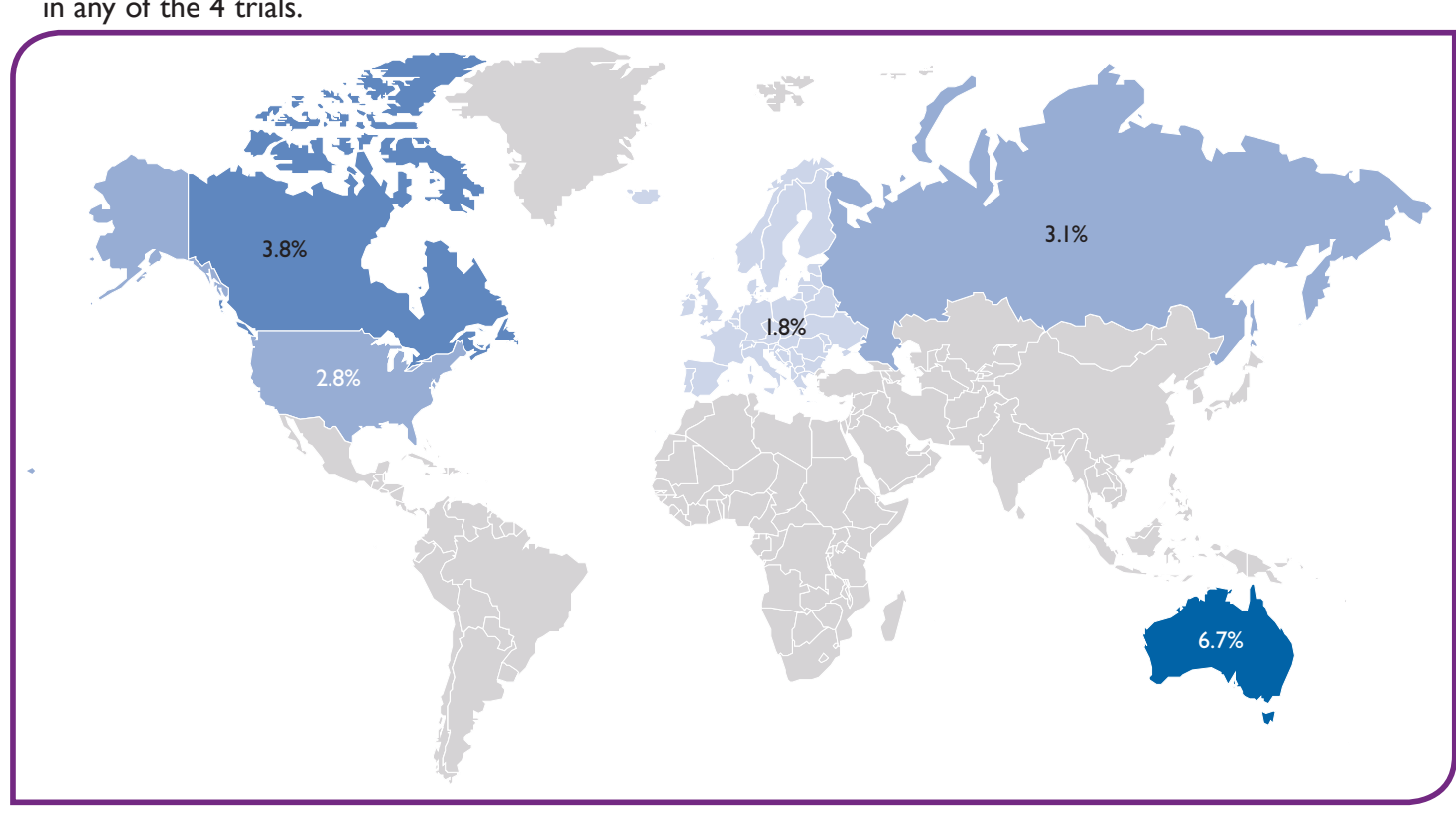

SIB, suicidal id
and Russia, 65

- Follow-up observation time-adjusted incidence rates of SIB events from the first dose of brodulumab through end of study were consistent across regions (Figure 4)

Figure 4. Follow-up observation time-adjusted incidence rates (per 100 patient-years) of SIB events from the first dose of brodalumab through end of study in patients from the long-term extension of any of the 4 trials.

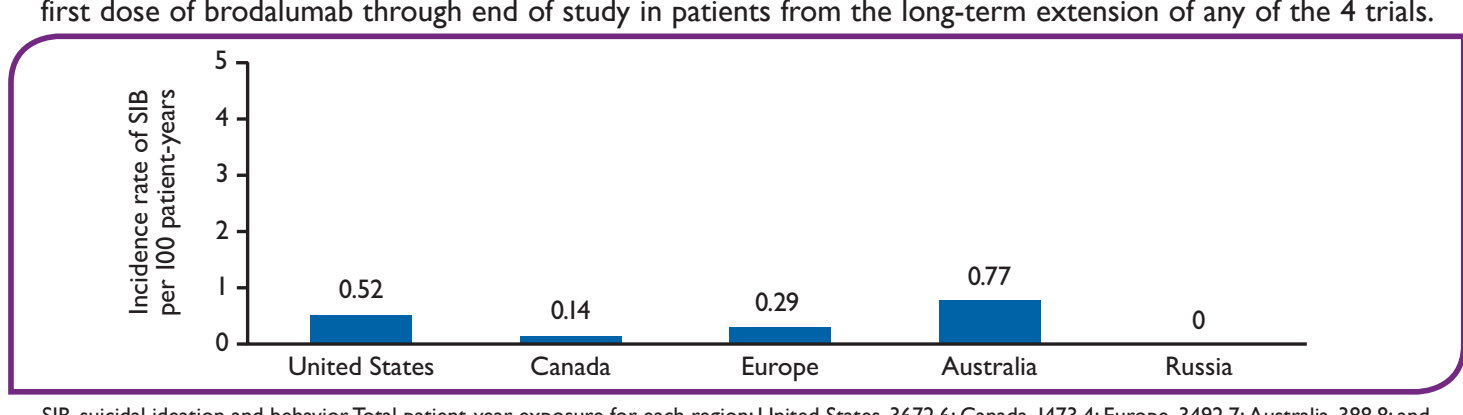

SIB, suicidal id
Russia, 134.4.

\section{CONCLUSIONS}

- Clinical trials of brodalumab reflected real-world populations of patients with moderate-to-severe psoriasis

- The patients in these 4 trials had baseline rates of depression and SIB that were consistent with those in other studies ${ }^{2}$

- The follow-up observation time-adjusted incidence rates (per 100 PY) of SIB events from the first dose of brodalumab through end of study were consistent across regions

\section{Amgen Inc.}

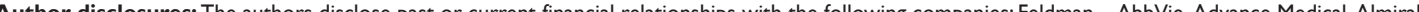
Anacor, Astellas, Baxter, Boehringer Ingelheim, Caremark, Celgene, Cosmederm Bioscience, Galderma, GlaxoSmithKline/Stiefel, Informa, Janssen, LEO Pharma Eli Lilly \& Co, Merck, Merz, Mylan, National Biological Corporation, National Psoriasis Foundation, Novan, Novartis, Parion, Pfizer, Qurient, Regeneron, Suncare Research, Taro, Up ToDate, Valeant Pharmaceuticals North America LLC, Gerson Lehrman, Guidepoint Global, www. DrScore.com, and Causa Research; Pharmaceuticals North America LLC.

References: I. Kurd SKK et al. Arch Dermatol. 2010;146:891-895. 2. Koo J et al. J Eur Acad Dermatol Venereol. 2017 [Epub ahead of print]. 3. Lebwohl M et al. N Engl J Med. 2015;373:1318-1328. 4. Papp KA et al. Br J Dermatol. 2016;175:273-286. 5. Papp KA et al. N Engl] Med. 2012;366:1181-I189. 